\title{
Regulação cardiovascular de idosos em ambientes com baixa oxigenação: uma revisão narrativa
}

\author{
Cardiovascular regulation of the elderly in low oxygenation environments: a narrative \\ review
}
Regulación cardiovascular de las personas mayores en ambientes de baja oxigenación: una revisión narrativa

Gabriel de Souza Zanini ${ }^{1,3 *}$, Bianca Fernandes ${ }^{1}$, Vanessa Teixeira do Amaral' ${ }^{1}$, Pedro Felisberto ${ }^{2}$.

\begin{abstract}
RESUMO
Objetivo: Descrever os efeitos adaptativos no controle cardiovascular em ambientes hipóxicos no público idoso, bem como, compreender os aspectos relacionados a essa exposição na Doença Pulmonar Obstrutiva crônica (DPOC). Revisão bibliográfica: Em elevadas altitudes é comum à instalação de hipóxia hipobárica, condição na qual há uma redução significante da saturação de oxigênio arterial, condição que induz os quimiorreceptores cardiovasculares a ajustes da ventilação pulmonar na busca do reestabelecimento dos níveis normais do suprimento de oxigênio. Contudo em indivíduos com DPOC expostos a ambientes de baixa oxigenação é observado que há um agravamento do quadro, visto uma sobrecarga nos sistemas periféricos, alterando as variáveis hemodinâmicas e estruturas funcionais do endotélio. Considerações finais: Os dados dos estudos analisados demonstraram que ambientes hipobáricos não alteram as capacidades adaptativas da regulação cardiovascular em idosos, porém, em indivíduos acometidos por DPOC, é observado uma piora no quadro geral da condição, ao passo que há uma perda na função endotelial.
\end{abstract}

Palavras-chave: Controle cardiovascular, Altitude, Envelhecimento, Doença pulmonar obstrutiva crônica, Hipóxia hipobárica.

\begin{abstract}
Objective: To describe the adaptive effects on cardiovascular control in hypoxic environments in older people, as well as to understand the aspects related to this exposure in chronic obstructive pulmonary disease (COPD). Bibliographic review: At high altitudes, the installation of hypobaric hypoxia is common, a condition in which there is a significant reduction in arterial oxygen saturation, a condition that induces cardiovascular chemoreceptors to adjust pulmonary ventilation in order to reestablish normal levels of oxygen supply. However, in individuals with COPD exposed to environments of low oxygenation, it is observed that there is a worsening of the condition, as there is an overload on the peripheral systems, changing the hemodynamic variables and functional structures of the endothelium. Final considerations: Data from the analyzed studies showed that hypobaric environments do not change the adaptive capacity of cardiovascular regulation in the elderly, however, in individuals affected by COPD, a worsening in the general condition of the condition is observed, while there is a loss in endothelial function.
\end{abstract}

Keywords: Cardiovascular control, Altitude, Aging, Chronic obstructive pulmonary disease, Hypobaric hypoxia.

\section{RESUMEN}

Objetivo: Describir los efectos adaptativos sobre el control cardiovascular en ambientes hipóxicos en personas mayores, así como comprender los aspectos relacionados con esta exposición en la enfermedad pulmonar obstructiva crónica (EPOC). Revisión bibliográfica: En altitudes elevadas es común la instalación de hipoxia hipobárica, condición en la que se produce una reducción significativa de la saturación arterial de

${ }^{1}$ Universidade Estadual Paulista (Unesp), Bauru - SP. *E-mail: gabriel.zanini@unesp.br

2 Universidade Estadual Paulista (Unesp), Presidente Prudente - SP.

${ }^{3}$ Faculdade Anhanguera, Bauru - SP. 
oxígeno, condición que induce a los quimiorreceptores cardiovasculares a ajustar la ventilación pulmonar para restablecer niveles normales de suministro de oxígeno. Sin embargo, en individuos con EPOC expuestos a ambientes de baja oxigenación, se observa que hay un empeoramiento de la condición, ya que existe una sobrecarga en los sistemas periféricos, cambiando las variables hemodinámicas y estructuras funcionales del endotelio. Consideraciones finales: Datos de los estudios analizados mostraron que los ambientes hipobáricos del no alteran la capacidad adaptativa de la regulación cardiovascular en el anciano, sin embargo, en los individuos afectados por EPOC se observa un empeoramiento en el estado general de la condición, mientras que existe una pérdida en el endotelio función.

Palabras clave: Control cardiovascular, Altitud, Envejecimiento, Enfermedad pulmonar obstructiva crónica, Hipoxia hipobárica.

\section{INTRODUÇÃO}

Diariamente pessoas no mundo todo (em especial sujeitos residentes de locais ao nível do mar com algum tipo de comorbidade pré-existente) expõem-se a regiões "hipóxicas" (caracterizadas por regiões/ambientes de grande altitude e/ou com baixa disponibilidade de oxigênio), para as mais diversas finalidades como moradia, esportes, viagens, pesquisa, tratamentos médicos e entre outros. No entanto, a exposição aguda e de longa duração em ambientes com essas características faz com que os sistemas orgânicos sejam induzidos a adaptar seus processos fisiológicos para que os sistemas perdurem de maneira satisfatória e menos nociva em ambientes com baixa oxigenação (GROTH A, et al., 2018; PARK HY, et al., 2019).

Estudos já demonstraram que exposições prolongadas a ambientes hipóxicos sem experiência ou preparação prévia podem gerar uma resposta fisiológica atípica e possivelmente danosa aos sistemas orgânicos, principalmente em públicos com idades mais avançadas (BÄRTSCH P e SWENSON ER, 2013; KINOSHITA A, et al., 2007; LHUISSIER FJ, et al., 2012). Isso se deve, pois, o processo de envelhecimento está atrelado com a disfunção de vários parâmetros fisiológicos, em especial, com a disfunção cardiopulmonar e cardiovascular (PARK HY, et al., 2019).

Com isso, a exposição prolongada a ambientes hipóxicos pode mediar possíveis complicações fisiológicas e fisiopatológicas em indivíduos com idades avançadas e/ou com algum tipo de disfunção cardiovascular ou cardiopulmonar, podendo ter estas complicações potencializadas mediante a presença de alguma destas disfunções em idade avançada (PARK HY, et al., 2019).

Devido às respostas fisiológicas induzidas por ambientes hipóxicos terem uma grande variação, pesquisadores têm procurado compreender como essas adaptações fisiológicas induzidas pela hipoxidade podem impactar na saúde global, principalmente dos sujeitos com as condições elencadas anteriormente. De todo modo, a exposição aguda ou crônica a ambientes hipóxicos desde o nacimento podem induzir elevados níveis de estresse para os sistemas fisiológicos, aumentando a predominância de condições clinicas como diabetes, hipertensão arterial e doença pulmonar obstrutiva crônica (MARTIN A, et al., 2018).

A partir do panorama apresentado anteriormente, este estudo teve como proposta descrever os principais efeitos adaptativos no controle cardiovascular, miocárdico e cardiorrespiratório em ambientes hipóxicos em idosos com algum tipo de disfunção cardiovascular e/ou cardiopulmonar. De modo que os desfechos tratados neste estudo possam ajudar orientar previamente os sujeitos com alguma destas disfunções durante a exposição a ambientes hipóxicos.

\section{REVISÃO BIBILIOGRÁFICA}

\section{Respostas Adaptativas do Sistema Cardiovascular e Cardiopulmonar a Ambientes Hipóxicos}

Em elevadas altitudes (superiores a 1000 metros acima do nível do mar) é comum à instalação da hipóxia hipobárica, esta condição ocorre devido à redução da pressão parcial de oxigênio atmosférico quando comparado ao nível do mar, que por sua vez, causa uma redução significante da saturação de oxigênio arterial. Essa condição induz os quimiorreceptores cardiovasculares ao ajuste da ventilação pulmonar para tentativa de reestabelecimento dos níveis normais de suprimento de oxigênio para os sistemas orgânicos (BERNARDI L, et al., 2001). 
Esse processo mediado pelos quimiorreceptores influencia diretamente a modulação da frequência cardíaca durante a exposição a situações de hipóxia, essa modulação ocorre de forma autonômica, a qual modifica o controle da frequência cardíaca por meio do barorreflexo cardíaco e da atividade simpática, assim como na modificação da atividade (simpática) vasoconstrictora (BERNARDI L, et al., 2001; HALLIWILL JR e MINSON CT, 2002; PARK MY, et al., 2019).

Em complemento, a partir do momento que a saturação de oxigênio arterial diminui, os sistemas orgânicos, em resposta, induzem a aumentos na ventilação mecânica com intuito de diminuir os efeitos estressores originários da hipoxidade, e também manter a integralidade do sistema de aporte energético (aeróbio) miocárdico. Neste sentido, em um estudo realizado por Magalhães J, et al. (2002) foi verificado que os valores ventilatórios podem ser de 2 a 5 vezes maiores em ambientes hipóxicos, sendo essa elevação responsável pelo funcionamento ajustadamente satisfatório dos sistemas orgânicos. Além disso, no mesmo estudo também foi observado que o fluxo sanguíneo apresenta tendência de se tornar mais seletivo em ambientes hipóxicos, no qual, os tecidos considerados vitais possuem prioridade de suprimento de sangue arterial.

Contudo, mesmo com a imposição dessas adaptações com intuito de manter um funcionamento orgânico satisfatório, estudos como Wilmore JH e Costil DL (2001) demonstram que o consumo máximo de oxigênio (VO2máx) não sofre reduções significativas até uma altitude de 1600 metros, no qual, a saturação de oxigênio apresenta tendência de decréscimo a partir de altitudes superiores a indicada, além disso, foi observado que a cada 1000 metros de incremento na altitude o valor de VO2máx apresenta tendência de decréscimo de $11 \%$. Portanto, esses achados ajudam a fomentar a necessidade do processo de adaptação prévia dos sistemas orgânicos para que o mesmo consiga desempenhar suas funções adequadamente quando exposto a ambientes hipóxicos.

Outro aspecto importante quando se trata da hipoxidade é a modulação do fluxo sanguíneo, a qual sofre alterações significativas quando se trata de grandes altitudes e/ou ambientes hipóxicos, pois, estas situações apresentam indução de perda de água corporal, a qual induz o aumento da viscosidade sanguínea. Com o sangue mais denso, o volume de ejeção apresenta redução, fazendo com que haja aumentos significantes no débito cardíaco e consequentemente, na frequência cardíaca, para tentar sanar temporariamente essa queda do volume de ejeção. Adicionalmente, aumentos na densidade sanguínea também ocasionam uma sobrecarga no coração devido à resistência aumentada exercida pelo sangue (mais denso) na parede dos vasos, podendo elevar o risco de lesão aterosclerótica e eventos cardíacos (SCHOENE RB, 2008; PARK MY, et al., 2019).

O breve panorama apresentado anteriormente foi evidenciado em um estudo de Lang M, et al. (2016), no qual foi possível identificar que indivíduos hipertensos (idade $52,2 \pm 9,7$ ) quando submetidos a um teste de caminhada de seis minutos em altitude elevada $(3260 \mathrm{~m})$, tiveram uma resposta de pressão arterial (PA) aumentada durante o repouso e, principalmente, logo após o teste, no qual, foi possível observar que a PA permaneceu elevada durante todo o período de recuperação. Esse achado chama a atenção, dado a aparente dificuldade do controle pressórico em pacientes hipertensos quando expostos a ambientes com baixa oxigenação, principalmente quando sujeitados a atividades leves que exigem uma resposta aguda satisfatória do sistema cardiovascular.

Em contrapartida, foi demonstrando que a exposição a grandes altitudes e/ou ambientes hipóxicos pode gerar benefícios a longo prazo, como evidenciado no estudo de Rostrup M (1998), no qual foi demonstrado que, o aumento da atividade simpática pode ser uma forma reflexa a um não aumento das concentrações de catecolaminas circulantes, mas, um incremento da estimulação de seus terminais nervosos. Contudo, a exposição prolongada a esse tipo de ambiente tende a promover uma diminuição significativa do volume plasmático e, simultaneamente, uma redução da frequência cardíaca máxima implicando por sua vez em uma redução do débito cardíaco máximo, o qual pode implicar de maneira direta no suprimento de sangue arterial para os tecidos, principalmente mediante a situações de moderada a alta intensidades (SIEBENMANN C, et al., 2017a; SIEBENMANN C, et al., 2017b)

No mesmo sentido, em estudo mais recente desenvolvido com idosos obesos $(66,5 \pm 0,8$ anos) submetidos a exercícios físicos em ambientes de grande altitude $(3000 \mathrm{~m})$, foi possível observar que a composição 
corporal, função pulmonar e variabilidade da frequência cardíaca melhoraram de maneira significativa, até mesmo superior quando comparados com idosos na mesma condição, porém, treinados em locais normóxicos. Desse modo, novos estudos devem ser realizados para verificar de maneira concreta os possíveis benefícios ou malefícios causados pela exposição de curto e longo prazo em regiões de grande hipoxidade (PARK HY, et al., 2019).

\section{Comportamento da pressão arterial e miocárdico frente à altitude em idosos com DPOC}

A Doença Pulmonar Obstrutiva Crônica (DPOC) caracteriza-se principalmente pela limitação do fluxo aéreo devido a sua obstrução crônica. A qual é resultante da disfunção da difusão gasosa entre as vias aéreas e os brônquios, juntamente com a inflamação e posteriormente, possível destruição do parênquima pulmonar (SCHWARZ El, et al., 2015). Tal condição é agravada durante a realização de esforços físicos ou com a permanência em ambientes hipóxicos como locais com mais de $1600 \mathrm{~m}$ de altitude devido à baixa pressão atmosférica de oxigênio (PARK HY, et al., 2019).

Esta condição quando não congênita, é mais comumente encontrada em sujeitos acima de 50 anos em decorrência de um acúmulo prospectivo de maus hábitos comportamentais como o uso ativo ou passivo de nicotina, permanência demasiada em ambientes com excesso de poluentes gasosos e entre outros (KOTAKI K, et al., 2019).

Como mencionado anteriormente, a DPOC é mais ocorrente em idosos, ao qual a junção destas duas condições está intimamente ligada com algumas comorbidades, em principal às cardiovasculares, com destaque para as alterações dos valores pressóricos, muitas vezes decorrentes da disfunção endotelial advinda do processo natural e irreversível do envelhecimento, com isso, sujeitos condicionados a tais características apresentam-se como mais responsivos as alterações do sistema cardiovascular induzidas por hipóxia, com destaque para os valores pressóricos (CHEN W, et al., 2015; RICHALET JP e LHUISSIER FJ, 2015).

Adicionalmente, a DPOC também parece estar associada com a regulação autonômica prejudicada do fluxo sanguíneo, desse modo, sujeitos condicionados as características elencadas anteriormente são excepcionalmente mais suscetíveis a alterações da regulação cardiovascular induzidas por hipóxia e/ou ambientes de grandes altitudes (SCHWARZ EI, et al., 2015).

Frente a isso, em um estudo randomizado e de campo identificou-se que, após um grupo de sujeitos com média de idade de 64 anos e moradoras de regiões de baixa altitude passarem 48 horas em um ambiente com mais de $2500 \mathrm{~m}$ de altitude, seus valores de frequência cardíaca e pressóricos foram demasiadamente alterados. Sendo que, nas primeiras 24 horas, os valores médios da PA diastólica matinal do grupo aumentaram em $+5,0 \mathrm{mmHg}$. O mesmo ocorreu com PA sistólica matinal média, porém, o aumento foi duas vezes maior $+10 \mathrm{mmHg}$. Adicionalmente, também foi identificado que 48 horas de permanência em ambiente de grande altitude foi o suficiente para causar diminuições significantes na sensibilidade barorreflexa $(-3,0$ $\mathrm{mseg} / \mathrm{mmHg}$ ) e um aumento progressivo e significativo na variabilidade cardíaca $(+1,8 \%)$ (SCHWARZ El, et al., 2015).

Nessa perspectiva, a hipertensão pulmonar também é uma das complicações característica da DPOC, a qual é linear a gravidade da inflamação e destruição do parênquima pulmonar. Esta condição específica em muitas das vezes vêm atrelada a complicações cardiovasculares, e neste caso em especial, com a disfunção ventricular (KUBOTA Y, et al., 2016; LICHTBLAU M, et al., 2019).

Frente a isso, em um estudo randomizado e de campo conduzido por Lichtblau M, et al. (2019) identificouse que, após um grupo de pessoas com mediana de idade de 66 anos (todos com DPOC e alguma disfunção cardiovascular e moradores de regiões de baixa altitude) passarem uma noite em um ambiente de $2590 \mathrm{~m}$ de altitude, foi suficiente para causar alterações nos parâmetros cardiovasculares. No qual, uma noite neste ambiente foi o suficiente para causar uma diminuição significante na pressão parcial de oxigênio $(9,0 \mathrm{kPa}$ para $6,6 \mathrm{kPa}$ ) juntamente com a diminuição de $7 \%$ da saturação de oxigênio no sangue arterial (de $94 \%$ para $87 \%$ ). A elevada altitude também causou alterações morfológicas na função miocárdica do ventrículo direito, ao qual houve um aumento na velocidade sistólica do pico de regurgitação tricúspide (TRV) assim como no gradiente de pressão tricúspide (TPG) (TRV de 2,4 m/s para 2,8 m/s e TPG de $23 \mathrm{~mm}$ HG par $32 \mathrm{~mm} \mathrm{Hg}$ ). 
A via de saída do ventrículo direito juntamente com sua área sistólica final e pressão atrial direita também aumentaram com a exposição à altitude (LICHTBLAU M, et al., 2019). Em complemento, todos os participantes do estudo possuíam função sistólica do ventrículo esquerdo normal a 490m de altitude. Porém, a subida dos participantes para um ambiente de $2590 \mathrm{~m}$ de altitude foi suficiente para causar um aumento significante da frequência cardíaca juntamente com um aumento da PA sistólica, além de um ligeiro incremento no diâmetro da raiz da aorta e uma ligeira redução da fração de ejeção do ventrículo esquerdo (LICHTBLAU M, et al., 2019).

Assim, pode-se dizer que sujeitos idosos e com DPOC são mais vulneráveis à exposição aguda a ambientes hipóxicos e/ou de grandes altitudes, no qual essa exposição age de maneira direta e negativa na regulação autonômica e cardiovascular, levando a aumentos clinicamente significativos dos valores pressóricos e de frequência cardíaca (SCHWARZ El, et al., 2015).

Essas alterações podem ser explicadas devido ao fato de os estímulos externos que orientam a regulação autonômica cardiovascular receberem uma cascata de reações adversas, já que, a escassez de oxigênio estimula os quimiorreceptores e consequentemente, os metaborreceptores, a uma interpretação equivocada de não suprimento da demanda metabólica, interferindo de maneira direta e sistêmica no controle cardiovascular durante o repouso. Em complemento, ambientes de grandes altitudes estão atrelados a baixas temperaturas, portanto, a exposição a esse tipo de local faz com que os termorreceptores ganhem mais destaque durante a regulação cardiovascular, ao qual também pode interferir na homeostase cardiovascular durante o repouso (AYME-DIETRICH E, et al., 2017).

Portanto, a exposição de idosos a ambientes hipóxicos leva a uma cascata de estímulos periféricos desproporcionais, os quais interferem de maneira direta na regulação cardiovascular central, podendo até sobrepor-se a ela, o que gera a desregulação do fluxo sanguíneo, e consequentemente, dos valores pressóricos e de frequência cardíaca no repouso ou durante atividades físicas leves. Em complemento, embora esses aumentos sejam uma resposta natural a tais condições, o estudo também identificou que tais consequências perpetuaram-se por mais tempo em sujeitos idosos e com DPOC quando comparados a sujeitos jovens sem DPOC expostos a mesma condição. Dessa maneira, a exposição a ambientes hipóxicos e/ou de grandes altitudes mostrou-se nociva e possivelmente deletéria a saúde cardiovascular de idosos com DPOC (SCHWARZ El, et al., 2015).

É fato que o processo de envelhecimento tornou-se um problema de saúde pública nos dias atuais, já que alguns fatores como psicossociais, culturais, medicinais e econômicos tem contribuído para o aumento da longevidade da humanidade, culminando na maior população da faixa etária idosa, que no ano de 2050, representará $16,7 \%$ da população mundial, com quase 1,6 bilhões idosos em todo o planeta (HE W, et al., 2016). Portanto, tal episódio necessita de atenção, uma vez que, concomitantemente ao processo de envelhecimento, há a deterioração dos tecidos e dos processos fisiológicos do corpo humano, que estão intimamente ligados com o processo inevitável do envelhecimento. Tal circunstância leva há um declínio das capacidades físicas, funcionais e fisiológicas, no qual alguns desses processos, interferem diretamente na regulação cardiovascular como o a disfunção da musculatura esquelética e cardiovascular (FAJEMIROYE JO, et al., 2018).

O processo de envelhecimento por si só já é um fator de risco para eventos cardiovasculares, no qual dentro deste processo, destaca-se o aumento da rigidez do endotélio vascular (SUN Z, 2015). O endotélio vascular possui papel direto na regulação do tônus vascular, coagulação, homeostase da circulação sanguínea, e mediação de substâncias vasodilatadoras, além de produzir respostas anti-inflamatórias, e ser o elo de ligação do sistema cardiovascular. Com o envelhecimento, todo esse sistema envolvido no controle da função endotelial sofre grande deterioração, levando ao quadro de disfunção endotelial, ao qual é inerente e comum na população idosa (RAJ V, et al., 2021).

Tal quadro também está intimamente associado com a ocorrência de eventos cardíacos e óbitos em idosos, sendo responsável por um grande percentual de óbitos neste público, de modo que tais circunstâncias podem ser agravadas quando há a exposição a ambientes hipóxicos ou com baixas concentrações de oxigênio (DE TORBAL A, et al., 2006; SANCHIS-GOMAR F, et al., 2016). 
Portanto, a exposição a estímulos hipóxicos pode gerar uma cascata de reações deletérias em sujeitos idosos, ao qual podem se agravar caso haja a presença de algum tipo de comorbidade. Já que, a exposição a ambientes hipóxicos causam desregulações no sistema central responsável pela regulação cardiovascular, assim, sobrecarregando os sistemas periféricos, gerando aumentos da frequência cardíaca de repouso, PA e variabilidade cardíaca, no qual, o incremento agudo destes fatores pode preceder eventos cardíacos e de maneira crônica, o óbito. Outro ponto importante a destacar-se é o fato de a exposição a ambientes hipóxicos gerar um estresse de cisalhamento hemodinâmico oscilatório, ao qual este processo é um fator de risco aterosclerótico, já que o aumento desse estresse induzido por hipóxia aumenta as chances de desenvolvimento de lesões na parede do endotélio (TREMBLAY JC, et al., 2017).

Adicionalmente, a exposição a estímulos hipóxicos também pode causar desregulações homeostáticas e metabólicas prejudiciais à saúde cardiovascular de idosos. Já que, para que haja a manutenção da homeostase corporal faz-se necessário contrações constantes e padronizadas de todas as musculaturas corporais, com destaque para musculatura cardíaca (GRASSI B, et al., 2015).

Para que a operação destes sistemas aconteça de maneira específica e eficiente, é fundamental a presença de oxigênio, portanto, a exposição prolongada a ambientes hipóxicos pode causar uma inversão do metabolismo responsável por suprir a demanda metabólica do processo de manutenção da homeostase, podendo levar a um aumento da produção de metabólitos e fadiga periférica, que quando em excesso, induzem a inflamação aguda de baixo grau, a qual estimula de maneira direta o aumento das chances de eventos cardíaco letais em sujeitos condicionados aos cenários apresentados anteriormente (CAO Z, et al., 2019; CUTLER BR, et al., 2018).

Em complemento, a disfunção endotelial também provoca a menor responsividade à vasodilatação mediada por óxido nítrico, podendo levar ao desenvolvimento de lesões ateroscleróticas e eventos cardíacos letais (BRUNO RM, et al., 2016; LANG M, et al., 2016).

Como já mencionado, a exposição a ambientes hipóxicos pode mediar o aumento de metabólitos e fadiga periférica, que em excesso, induzem a deterioração da integridade cardiovascular, no entanto, muitos dos sujeitos enquadrados nas condições apresentadas anteriormente desconhecem os potenciais riscos da exposição a ambientes hipóxicos, desse modo, recomenda-se que a exposição de sujeitos com estas condicionantes a ambientes hipóxicos seja comedida, principalmente se houver a presença de comorbidades agravantes. E se possível, que tal exposição seja feita após a intervenção e liberação de um médico especialista, ou, após a familiarização fisiológica do sujeito as condições ao qual ele será exposto, de modo que as consequências desta exposição não sejam tão graves a saúde cardiovascular (BRUNO RM, et al., 2016; LANG M, et al., 2016).

\section{CONSIDERAÇÕES FINAIS}

Parâmetros cardiovasculares de idosos não apresentam alterações quando expostos a ambientes normobáricos. Todavia, tal panorama sofre grandes alterações mediante a exposição à hipoxidade. Com isso, conclui-se que, a exposição a ambientes hipóxicos pode influenciar de maneira negativa na homeostase hemodinâmica e mecânica cardíaca de idosos. No qual, tais circunstâncias são agravadas mediante a presença da DPOC. Porém, mesmo com os dados apresentados, não é possível afirmar que tais alterações são exclusivamente derivadas da exposição à hipoxidade, já que, a DPOC pode atuar como um possível fator confundidor. Por fim, destaca-se que, o estudo contribuiu diretamente para a elucidação de uma temática importante, porém, que ainda possui escassez de estudos, indicando um campo vasto para estudos futuros.

\section{REFERÊNCIAS}

1. AYME-DIETRICH E, et al. Cardiovascular remodeling and the peripheral serotonergic system. Archives of Cardiovascular Diseases, 2017; 110(1): 51-59.

2. BÄRTSCH P, SWENSON ER. Acute High-Altitude Illnesses. New England Journal of Medicine 2013; 368(24): 22942302. 
3. BERNARDI L. et al. Respiratory and cardiovascular adaptations to progressive hypoxia. European Heart Journal, 2001; 22(10): 879-886.

4. BRUNO RM, et al. Vascular adaptation to extreme conditions: The role of hypoxia. Artery Research, 2016; 14: 15-21.

5. CAO Z, et al. An Integrated Analysis of Metabolites, Peptides, and Inflammation Biomarkers for Assessment of Preanalytical Variability of Human Plasma. Journal of Proteome Research, 2019: 18(6): 2411-2421.

6. CHEN W, et al. Risk of cardiovascular comorbidity in patients with chronic obstructive pulmonary disease: a systematic review and meta-analysis. The Lancet Respiratory Medicine, 2015; 3(8): 631-639.

7. CUTLER BR, et al. Blueberry metabolites restore cell surface glycosaminoglycans and attenuate endothelial inflammation in diabetic human aortic endothelial cells. International Journal of Cardiology, 2018; 261: 155-158.

8. DE TORBAL A, et al. Incidence of recognized and unrecognized myocardial infarction in men and women aged 55 and older: the Rotterdam Study. European Heart Journal, 2006, 27(6): 729-736.

9. FAJEMIROYE JO, et al. Aging-Induced Biological Changes and Cardiovascular Diseases. BioMed Research International, 2018; 2018: 7156435.

10. GRASSI B, et al. Skeletal Muscle Fatigue and Decreased Efficiency: Two Sides of the Same Coin? Exercise and Sport Sciences Reviews, 2015; 43(2).

11. HALLIWILL JR, MINSON CT. Effect of hypoxia on arterial baroreflex control of heart rate and muscle sympathetic nerve activity in humans. Journal of Applied Physiology, 2002; 93(3): 857-864.

12. HE W, et al. Internation Population Reports, P95/16-1, An Aging World: 2015, U.S Government Publishing Office, Washington, DC, 2016, Disponível em: https://www.census.gov/content/dam/Census/library/publications/2016/demo/p95-16-1.pdf.

13. KINOSHITA A, et al. Roles of Hemoglobin Allostery in Hypoxia-induced Metabolic Alterations in Erythrocytes: simulation and its verification by metabolome analysis*. Journal of Biological Chemistry, 2007; 282(14): 10731-10741.

14. KOTAKI K, et al. Trends in the prevalence of COPD in elderly individuals in an air-polluted city in Japan: a crosssectional study. International journal of chronic obstructive pulmonary disease, 2019; 14(1): 791.

15. KUBOTA Y, et al. COPD advances in left ventricular diastolic dysfunction. International journal of chronic obstructive pulmonary disease, 2016; 11(1): 649-655.

16. LANG M, et al. Blood pressure response to six-minute walk test in hypertensive subjects exposed to high altitude: effects of antihypertensive combination treatment. International Journal of Cardiology, 2016; 219(1): 27-32.

17. LHUISSIER FJ, et al. Ageing and cardiorespiratory response to hypoxia. The Journal of Physiology, 2012; 590(21): 5461-5474.

18. LICHTBLAU M, et al. Right and Left Heart Function in Lowlanders with COPD at Altitude: Data from a Randomized Study. Respiration, 2019; 97(2): 125-134.

19. MAGALHÃES J, et al. O desafio da altitude. Uma perspectiva fisiológica. Revista Portuguesa de Ciências do Desporto, 2002; 2(4): 81-91.

20. MARTIN A, et al. Preterm birth and oxidative stress: Effects of acute physical exercise and hypoxia physiological responses. Redox Biology, 2018; 17(1): 315-322.

21. PARK HY, et al. Twelve weeks of exercise modality in hypoxia enhances health-related function in obese older Korean men: A randomized controlled trial. Geriatrics \& Gerontology International, 2019; 19(4): 311-316.

22. RAJ V, et al. Avaliação da Senescência de Células Sanguíneas Mononucleares Periféricas e na Disfunção Endotelial entre Adultos com Alto Risco Cardiovascular. Arquivos Brasileiros de Cardiologia, 2021; (116): 37-47.

23. RICHALET JP, LHUISSIER FJ. Aging, Tolerance to High Altitude, and Cardiorespiratory Response to Hypoxia. High Altitude Medicine \& Biology, 2015; 16(2): 117-124.

24. ROSTRUP M. Catecholamines, hypoxia and high altitude. Acta Physiologica Scandinavica, 1998; 162(3): 389-399.

25. SANCHIS-GOMAR F, et al. Epidemiology of coronary heart disease and acute coronary syndrome. Annals of translational medicine, 2016; 13(4): 256-264.

26. SCHOENE RB. IIInesses at High Altitude. Chest, 2008; 134(2):402-416.

27. SCHWARZ E, et al. Blood pressure response to altitude exposure in patients with COPD. European Respiratory Journal, 2015; 46(59): 2309-2315.

28. SIEBENMANN C, et al. Regulation of blood volume in lowlanders exposed to high altitude. Journal of Applied Physiology, 2017a; 123(4): 957-966.

29. SIEBENMANN C, et al. Parasympathetic withdrawal increases heart rate after 2 weeks at $3454 \mathrm{~m}$ altitude. The Journal of Physiology, 2017b; 595(5): 1619-1626.

30. SUN Z. Aging, Arterial Stiffness, and Hypertension. Hypertension, 2015; 65(2): 252-256.

31. TREMBLAY JC, et al. Oscillatory shear stress, flow-mediated dilatation, and circulating microparticles at sea level and high altitude. Atherosclerosis, 2017: 256(1): 115-122.

32. WILMORE JH, COSTILL DL. Physical energy: fuel metabolism. Nutrition reviews, 2001; 59(1): 13-27. 